LUNG CANCER

\title{
High magnification bronchovideoscopy combined with narrow band imaging could detect capillary loops of angiogenic squamous dysplasia in heavy smokers at high risk for lung cancer
}

\author{
K Shibuya, H Hoshino, M Chiyo, A lyoda, S Yoshida, Y Sekine, T lizasa, Y Saitoh, M Baba, \\ K Hiroshima, H Ohwada, T Fujisawa
}

Thorax 2003;58:989-995

See end of article for authors' affiliations

Correspondence to: Dr T Fujisawa, Department of Thoracic Surgery, Graduate School of Medicine, Chiba University, 1-8-1 Inohana, Chuo-ku, Chiba 2608670, Japan; fujisawa@ med.m.chiba-u.ac.jp
Background: We investigated the use of high magnification bronchovideoscopy combined with narrow band imaging (NBI) for the detailed examination of angiogenic squamous dysplasia (ASD). This was carried out in relation to bronchial vascular patterns with abnormal mucosal fluorescence in heavy smokers at high risk for lung cancer.

Methods: Forty eight patients with sputum cytology specimens suspicious or positive for malignancy were entered into the study. Conventional white light and fluorescence bronchoscopic examination was first performed. Observations by high magnification bronchovideoscopy with conventional white light were made primarily at sites of abnormal fluorescence, and then repeated with NBI light to examine microvascular networks in the bronchial mucosa. Spectral features on the RGB (Red/Green/Blue) sequential videoscope system were changed from the conventional RGB broadband filter to the new NBI filter. The wavelength ranges of the new NBI filter were B1: 400-430 nm, B2: 420-470 nm, and G: 560$590 \mathrm{~nm}$. ASD tissues were also examined using a confocal laser scanning microscope equipped with argon-krypton (488 $\mathrm{nm})$ and argon $(514 \mathrm{~nm}$ ) laser sources.

Results: The microvessels, vascular networks of various grades, and dotted vessels in ASD tissues were clearly observed in NBI-B1 images. Diameters of the dotted vessels visible on NBI-B1 images agreed with the diameters of ASD capillary blood vessels diagnosed by pathological examination. Capillary blood vessels were also clearly visualised by green fluorescence by confocal laser scanning microscopy. There was a significant association between the frequency of dotted vessels by $\mathrm{NBI}-\mathrm{Bl}$ imaging and tissues confirmed as ASD pathologically $(p=0.002)$.

Conclusions: High magnification bronchovideoscopy combined with NBI was useful in the detection of capillary blood vessels in ASD lesions at sites of abnormal fluorescence. This may enable the discrimination between ASD and another pre-invasive bronchial lesion.
$\mathrm{T}$ he use of fluorescence bronchoscopy in heavy smokers at high risk for lung cancer has increased the recognition of cases of bronchial squamous dysplasia. ${ }^{1-4}$ Increased thickening of the bronchial epithelium, increased vessel growth and a loss of fluorophore concentration are thought to be related to the appearance of areas of abnormal fluorescence. This may explain why many observers have found inflammation in mucosa with abnormal fluorescence. ${ }^{5}$ Recently, a new morphological entity called angiogenic squamous dysplasia (ASD) was identified in the large central airways by fluorescence bronchoscopy, whereby collections of capillary blood vessels were closely juxtaposed to, and projected into, dysplastic bronchial epithelium. ${ }^{6}{ }^{7}$ Increased vessel density in the submucosa is often present in bronchial squamous dysplasia, ${ }^{89}$ indicating that angiogenesis is a relatively early event during lung cancer pathogenesis. ${ }^{10}$ Capillary blood vessels in the dysplastic epithelium in ASD also show evidence of angiogenesis by histopathology, such that it is difficult to distinguish ASD from conventional squamous dysplasia (i.e. without capillary blood vessels in bronchial squamous dysplasia) using white light bronchoscopy as well as fluorescence bronchoscopy. However, it remains to be determined whether or not ASD is at greater risk for progression than conventional squamous dysplasia. $^{10}$
Recently, we developed a direct viewing high magnification bronchovideoscopy system with the aim of making detailed examination of dysplasia in the bronchial mucosa of patients exhibiting abnormal mucosal fluorescence. ${ }^{11}$ The high magnification bronchovideoscope has an outer diameter of only $6 \mathrm{~mm}$, which can be easily inserted into the tracheobronchial tree. Microvessels in the bronchial submucosa can be observed on a 14-inch TV monitor at a high magnification of up to $110 \times$. Our previous report showed that many cases of squamous dysplasia have vascular patterns of increased vessel growth and complex networks of tortuous vessels in the bronchial subepithelium. ${ }^{11}$ Thus, using the in vivo examination technique of high magnification bronchovideoscopy, it was possible to demonstrate angiogenesis in the submucosa of bronchial dysplasia patients that could not be recognised by conventional white light bronchoscopy.

In the present study, we investigated the ability of a high magnification bronchovideoscopy system combined with narrow band imaging (NBI) to detect capillary blood vessels in the dysplastic bronchial epithelium of heavy smokers at high risk for lung cancer. We changed the spectral features of

Abbreviations: $\mathrm{NBI}$, narrow band imaging; $\mathrm{ASD}$, angiogenic squamous dysplasia; RGB, Red/Green/Blue 
the RGB sequential videoscope system from the conventional RGB (Red/Green/Blue) broadband filter to the new NBI filter in order to allow focused and detailed observation of bronchial vascular patterns. The wavelength ranges of the new NBI filter included the NBI-Blue 1 (NBI-Bl) filter at $400-430 \mathrm{~nm}$, which covered the $410 \mathrm{~nm}$ absorption wavelengths for haemoglobin, and therefore suggested the possibility of more accurate detection of vessel structures.

\section{METHODS}

\section{Narrow band imaging}

The conventional RGB sequential videoscope system has a xenon lamp and rotation disk with 3 RGB optical filters. The rotation disk and monochrome CCD are synchronised and 3 band images are generated sequentially. Colour images can be synthesised using 3 band images by the video processor (fig 1A). Narrow band imaging, developed in conjunction with the Olympus Optical Corp., Tokyo, Japan, is a novel system that can be used to observe microvessel structure using a new narrow banding filter on an RGB sequential videoscope system instead of the conventional RGB broadband filter. Wavelength ranges of the new NBI filter were $\mathrm{Bl}$ : $400-430 \mathrm{~nm}, \mathrm{~B} 2: 420-470 \mathrm{~nm}$, and G: $560-590 \mathrm{~nm}$. In contrast, the range of wavelengths in the conventional RGB broadband filter were B: $400-500 \mathrm{~nm}, \mathrm{G}: 500-600 \mathrm{~nm}$, and R: 600-700 nm (fig 1B). Tissue optical absorption properties and scattering properties are strongly wavelength dependent, ${ }^{12}$ such that blue light, which has a shorter wavelength than visible light, typically reaches into shallow surfaces. ${ }^{13}$ The main chromophore in bronchial tissues in the visible wavelength range is haemoglobin, which has a maximum absorptive wavelength near $415 \mathrm{~nm},{ }^{14}{ }^{15}$ and is within the wavelength range for NBI-Bl. Thus, it was expected that the NBI-Bl filter would detect blood vessel structures more
A

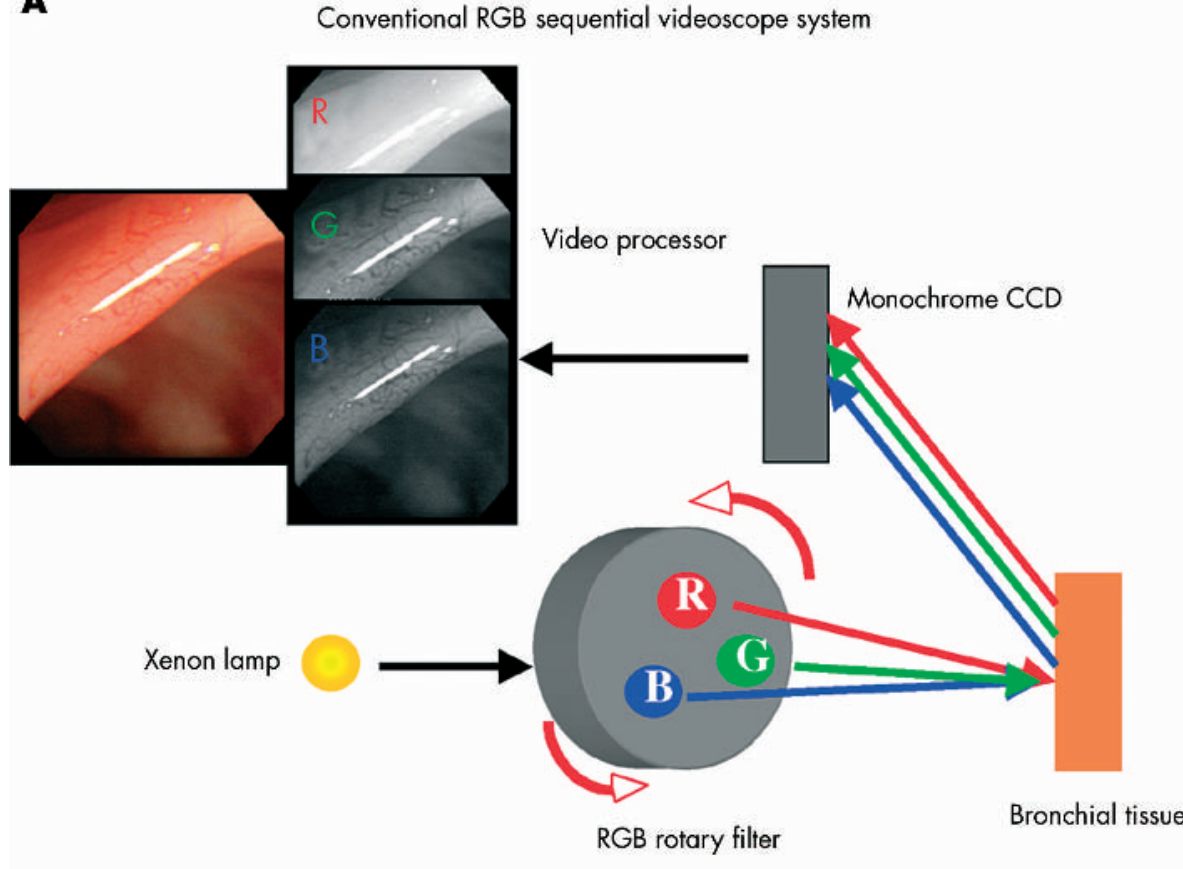

B

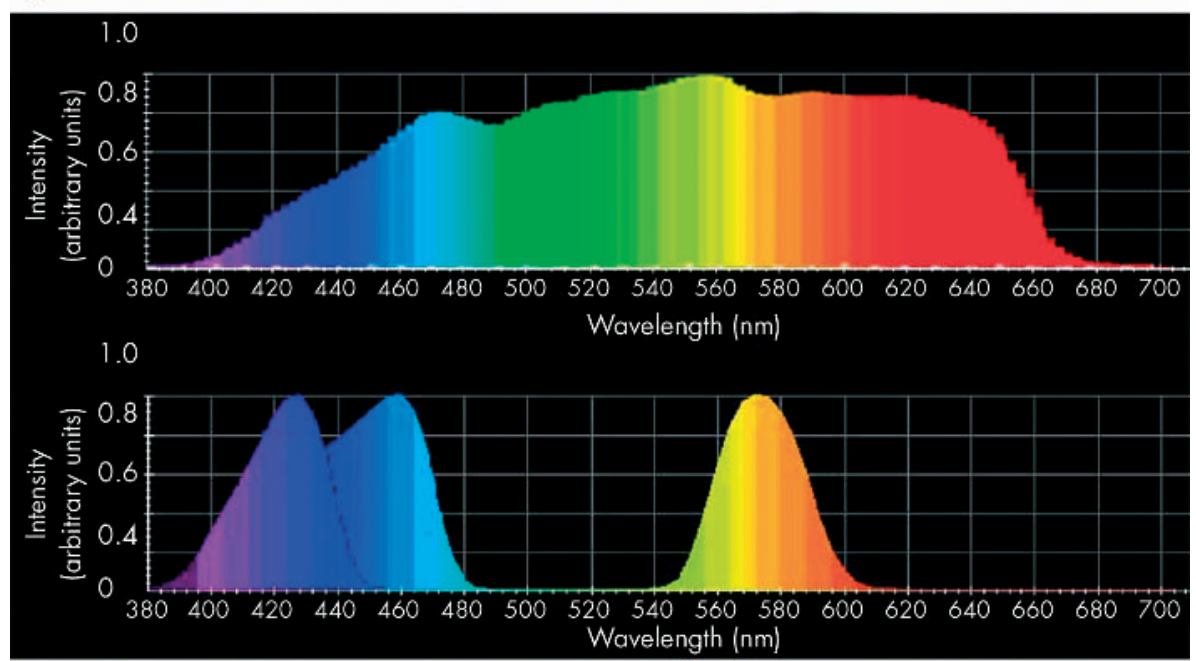

Figure 1 Conventional RGB system and the new NBI (narrow band imaging) system. The conventional RGB sequential videoscope system has a xenon lamp and rotation disk with 3 RGB optical filters. The rotation disk and monochrome CCD are synchronised and 3 band images are generated sequentially. Colour images can be synthesised using 3 band images by the video processor (fig 1A). Narrow band imaging (NBI) is a novel system that can be used to observe microvessel structure using a new narrow banding filter on an RGB sequential videoscope system instead of the conventional RGB broadband filter. Wavelength ranges of the new NBI filter were: $\mathrm{BI}=400-$ $430 \mathrm{~nm}, \mathrm{~B} 2=420-470 \mathrm{~nm}$, and $\mathrm{G}=560-590 \mathrm{~nm}$. In contrast, the range of wavelengths in the conventional RGB broadband filter were: $B=400$ $500 \mathrm{~nm}, \mathrm{G}=500-600 \mathrm{~nm}$, and $\mathrm{R}=600-700 \mathrm{~nm}$. $\mathrm{X}=$ Wavelength, $Y=$ Light Intensity (fig 1B).

New NBI filter 
accurately than other filters. When conventional RGB broadband light is delivered through an endoscope onto a tissue surface, some of the light is reflected from the tissue, some is scattered or absorbed within the tissue, and little light is detected to form an image on the television monitors. However, narrow band light delivered onto the same surface shows less scattering and makes it possible to show clearer television monitor images.

\section{High magnification bronchovideoscopy}

A high magnification bronchovideoscope (XBF 200HM2) is the combination of two systems, a video observation system for high magnification observation and a fibre observation system for orientation of the bronchoscope tip, as previously described in detail. ${ }^{11}$ While conventional white light bronchovideoscopy observed only increased redness and local swelling, the high magnification bronchovideoscope enabled the vascular networks with increased vessel growth and complex networks of tortuous vessels of various sizes in the bronchial mucosa to be visualised (fig 2).

\section{Study population and bronchoscopic procedure}

The Ethics Committees of the Graduate School of Medicine, Chiba University, approved all protocols and procedures. Forty eight patients with sputum cytology specimens suspicious or positive for malignancy seen at the Department of Thoracic Surgery, Graduate School of Medicine, Chiba University in Chiba, from December 2000 to April 2002, were entered into the study. A mass screening of sputum cytological examinations was carried out to identify cases at high risk for lung cancer. The high risk group included those 50 years or older with 30 or more pack years, and those 40 years or older who had bloody sputum within the past 6 months. The patients consisted of 46 males and 2 females and ranged in age from 42 to 77 years (mean 66 years). Smoking history in pack-years ranged from 25 to 155 (mean 54). Forty one of the patients were current smokers and 7 were ex-smokers. Conventional white light bronchoscopy (BF-240, Olympus Optical Corp, Tokyo, Japan) was first performed under local anaesthesia with sedation by intravenous midazolam injection and $\mathrm{O}_{2}$ inhalation. Fluorescence bronchoscopy examination was performed using the LIFE lung-system (Xillix LIFE-Lung Fluorescence Endoscopy System, Xillix Technologies Corp, Richmond, BC, Canada). Observations by high magnification bronchovideoscopy with conventional white light were made primarily at sites of abnormal fluorescence established by fluorescence bronchoscopy, and then repeated with NBI light to examine microvascular networks in the bronchial mucosa. Bronchial

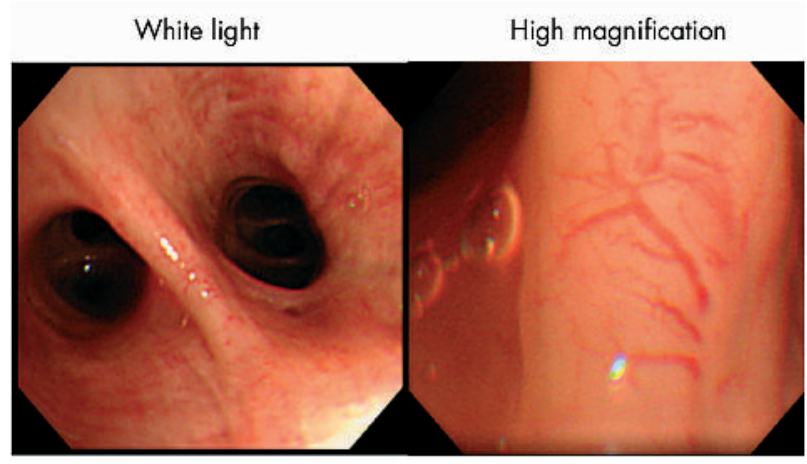

Figure 2 Conventional white light bronchovideoscopy and high magnification bronchovideoscopy of the bronchial mucosa. While conventional white light bronchovideoscopy observed only redness and swelling, high magnification bronchovideoscopy facilitated the observation of complex vascular networks of various sizes in the bronchial mucosa. biopsy specimens for pathological examination were obtained from all abnormal areas examined. Images obtained by high magnification bronchovideoscopy were examined and compared with pathological diagnoses from bronchial biopsy specimens. All participants provided written informed consent before enrolment into the study.

\section{High magnification bronchovideoscopy combined with NBI of the bronchial mucosa}

High magnification bronchovideoscopy combined with NBI enabled the microvascular structures in the bronchial mucosa to be visualised more accurately. Typical high magnification bronchoscopic findings from conventional RGB and NBI are shown (fig 3). Compared to conventional RGB images, NBI-B1/NBI-B2/NBI-G images provided more accurate images of various grades of microvessels, particularly using NBI-B1. Thus, using our new NBI system, it was possible to discern microvessel structures that could not be seen by means of conventional broadband systems, especially when using $\mathrm{Bl}$ images. In the NBI-Bl images, the vessels were seen to be clearly as dotted among the complex networks of tortuous vessels of various sizes, which we termed the "dotted vessels". The observation of vessels, regardless of whether they were seen as dotted or not, were without knowledge of the presence or absence of the pathological diagnosis of angiogenic squamous dysplasia.

\section{Histopathological analysis}

Biopsy specimens were fixed in $10 \%$ neutral formalin, embedded in paraffin, and stained with hematoxylin-eosin for histological study. Biopsy slides were first evaluated by a pathologist in our department, and then all slides were reviewed by two expert pulmonary pathologists in our department ( $\mathrm{KH}$ and $\mathrm{HO}$ ). Bronchial squamous dysplasia was diagnosed according to recent World Health Organization criteria. ${ }^{16}$ Angiogenic squamous dysplasia (fig 4) was characterised by microscopic projections into the bronchial lumen surface by squamous bronchial epithelium exhibiting variable degrees of squamous dysplasia. ${ }^{6}$ Bronchitis was defined as the condition in which the wall of the airways is infiltrated by lymphocytes and its blood vessels are congested.

\section{Confocal laser scanning microscope observation}

Bronchial biopsy specimens fixed overnight in 10\% neutral formalin were rinsed in PBS and applied to indirect immunofluorescence. Specimens were incubated overnight at $4^{\circ} \mathrm{C}$ with a mouse monoclonal IgG antibody against CD34 (Immunotech, Marseille, France) (1:10 dilution) as the primary antibody. After several washes, the specimens were incubated with fluorescent dye-labelled goat anti-mouse IgG, Alexa Fluor $488^{\circledR}$ (Molecular Probes, Inc. Eugene, Oregon, USA) (1:500 dilution) as the secondary antibody for $60 \mathrm{~min}$ at room temperature in the dark. Nuclear counterstaining was done for $15 \mathrm{~min}$ at room temperature with propidium iodide. Bronchial specimens were then examined using a confocal laser scanning microscope (BioRad; MRC-600) equipped with argon-krypton (488 $\mathrm{nm})$ and argon $(514 \mathrm{~nm})$ laser sources. In the resulting images, green fluorescence represented CD34, while cell nuclei appeared orange in colour.

Calculation of dotted vessel diameter among various grades of microvessels seen by high magnification bronchovideoscopy combined with NBI ${ }^{11}$

Using known charts, magnification on a 14-inch television monitor was calculated. When $\mathrm{L}=$ actual size of the vessel targeted, $\mathrm{K}$ (conversion factor) $=9.1 \times 10^{2} \mu \mathrm{m} /$ pixel, D (distance between two points on Adobe Photoshop 6.0) 

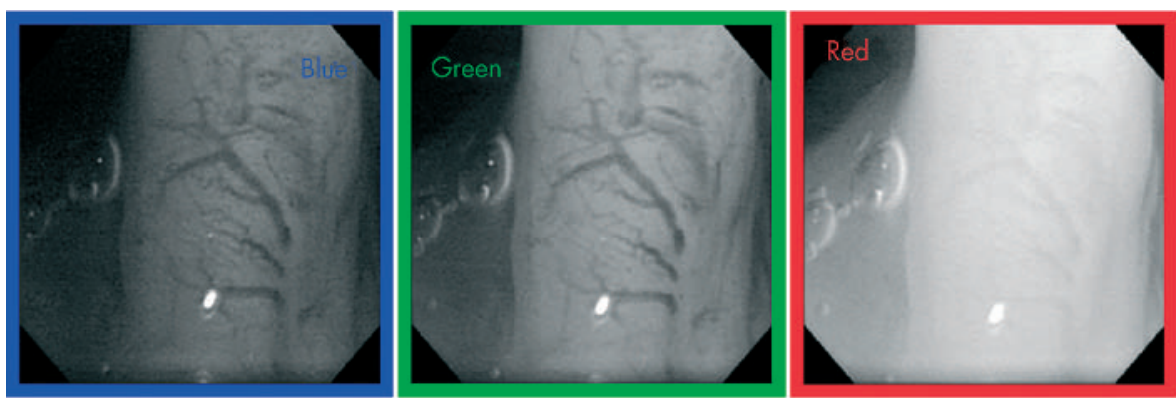

Figure 3 High magnification bronchovideoscopy in both conventional RGB and $\mathrm{NBI}$ of the bronchial mucosa. Typical high magnification bronchoscopic findings with conventional RGB or NBI are shown. Compared with conventional RGB images, NBI-B1/B2/G images could accurately detect various grades of micro vessels, especially NBI-B 1 imaging.

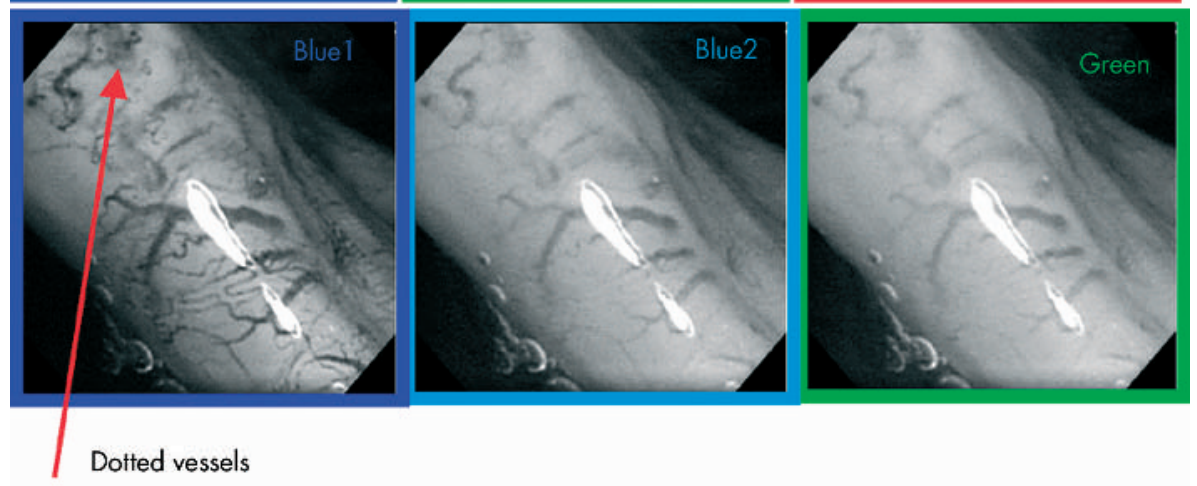

dysplasia. Of the 28 bronchial squamous dysplasia speci-

pixel $=\sqrt{ }\left\{(\mathrm{X} 1-\mathrm{X} 2)^{2}+(\mathrm{Y} 1-\mathrm{Y} 2)^{2}\right\}$, and $\mathrm{M}=$ Magnification, $\mathrm{L}$ is expressed as equalling $\mathrm{K} \times \mathrm{D} / \mathrm{M}$.

\section{Calculation of diameter of capillary blood vessels in ASD ${ }^{11}$}

Using an Image Cytometry (CAS 200) Cell Analysis System (Becton Dickenson, San Jose, Ca, USA) Micrometer Program, we calculated the diameter of the capillary blood vessels in ASD identified by histology using a haematoxylin/eosin stained biopsy slide.

\section{Statistical analysis}

Frequency analysis was performed with Fisher's exact probability test, with $\mathrm{p}$ values less than 0.05 considered statistically significant. Calculations were performed using the statistical software package Stat View (SAS Inc, Cary, NC, USA).

\section{RESULTS}

Pathological diagnoses of biopsy specimens

Biopsy specimens from 67 abnormal fluorescence sites were diagnosed as 39 bronchitis and 28 bronchial squamous

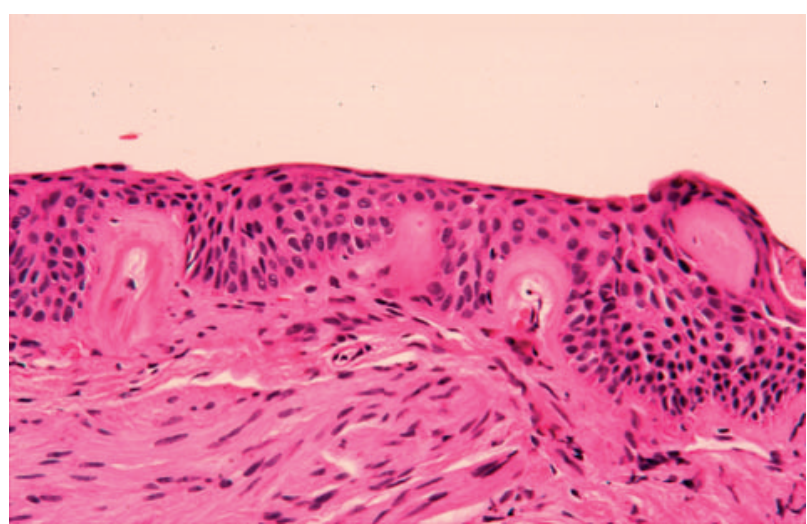

Figure 4 Photomicrograph of angiogenic squamous dysplasia. Collections of capillary sized blood vessels closely juxtaposed to, and projecting into, dysplastic bronchial epithelium. mens, 15 lesions demonstrated morphological changes characteristic of ASD.

\section{High magnification bronchovideoscopy combined with NBI of the bronchial mucosa}

Figure 5 shows four different bronchoscopic findings (white light bronchoscopy, fluorescence bronchoscopy, high magnification bronchovideoscopy and high magnification bronchovideoscopy with NBI-BI) at the right basal bronchus of a patient with sputum cytology suspicious for malignancy. Redness and swelling at the bifurcation of RT B8 and B9+10 was observed under white light examination, and an abnormal reddish brown area was identified by fluorescence examination. Increased vessel growth and complex networks of tortuous vessels were observed at sites of abnormal fluorescence by high magnification bronchovideoscopy with conventional RGB broadband filter. In the NBI-Bl images, the vessels were seen to be clearly dotted among the complex networks of tortuous vessels, which we termed the "dotted vessels". The pathological diagnosis of the specimen under biopsy showed ASD.

\section{Comparison between dotted vessels observed by high magnification bronchovideoscopy using $\mathrm{NBI}-\mathrm{B} 1$ and capillary sized blood vessels projecting into dysplastic epithelium (ASD) observed microscopically}

Using Adobe Photoshop 6.0, vessels observed as dots by high magnification bronchovideoscopy using NBI-Bl were calculated as $\mathrm{Ll}=53 \mu \mathrm{m}, \mathrm{L} 2=40 \mu \mathrm{m}, \mathrm{L} 3=25 \mu \mathrm{m}$ and $\mathrm{L} 4=48 \mu \mathrm{m}$. Capillary blood vessel diameters in ASD were calculated by Image Cytometry (CAS 200) Cell Analysis System as $\mathrm{C} 1=62.5 \mu \mathrm{m}, \mathrm{C} 2=50 \mu \mathrm{m}$, and C3 $=35 \mu \mathrm{m}$. Thus, the actual diameters, as seen in the histopathological samples, matched the vessel diameters seen by high magnification bronchovideoscopy (fig 6).

\section{Dual immunolabelling for confocal laser scanning microscopy}

Using a confocal laser scanning microscope, capillary blood vessels were visualised clearly as green fluorescence and the 


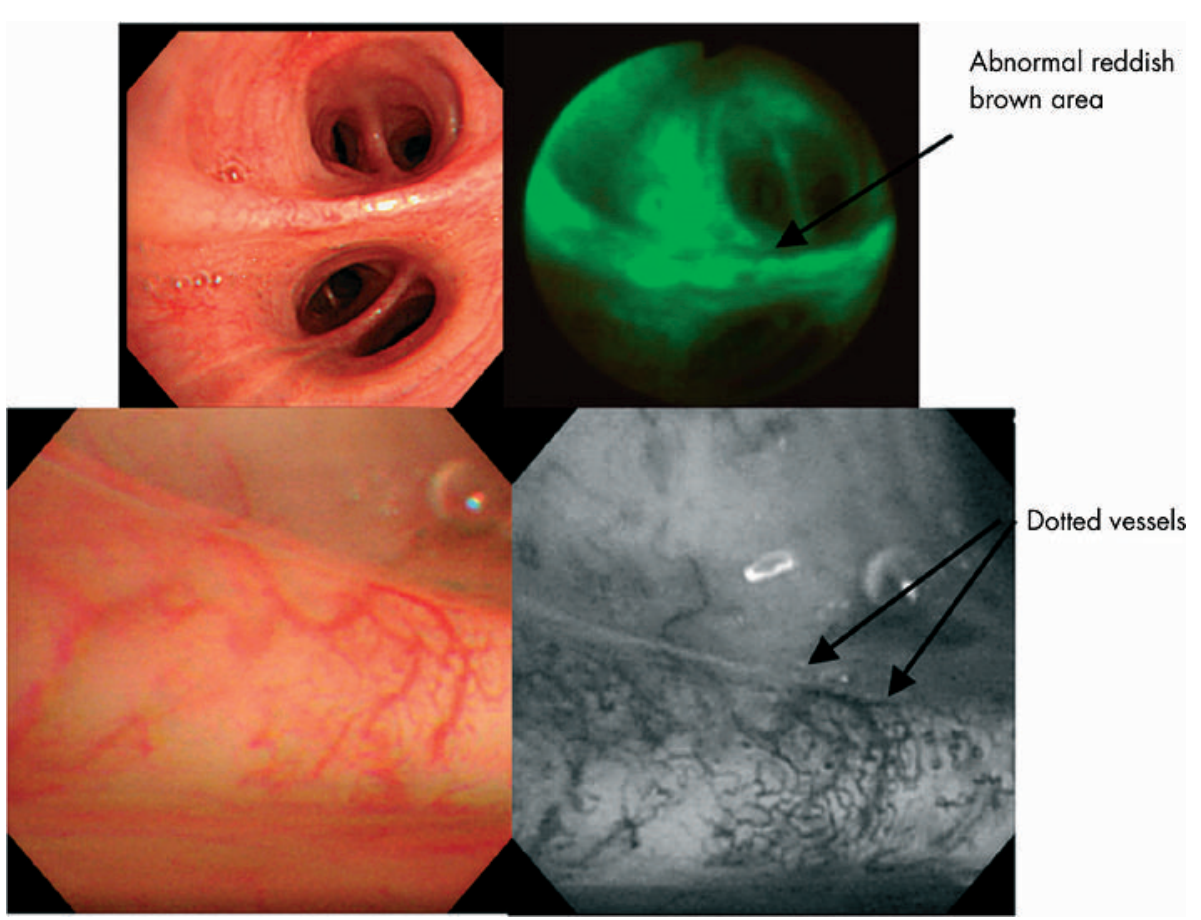

Figure 5 High magnification

bronchovideoscopy combined with NBI of the bronchial mucosa at sites of abnormal fluorescence. Some dotted vessels, in addition to increased vessel growth and complex networks of tortuous vessels, are visible on an $\mathrm{NBI}-\mathrm{B} 1$ image. The pathological diagnosis of the specimen under biopsy was angiogenic squamous dysplasia.

nuclei of dysplastic epithelium appeared orange in colour. We evaluated capillary loops in dysplastic epithelium to determine the validity of the dotted vessels detected by high magnification bronchovideoscopy combined with NBI-Bl imaging (fig 7).

\section{Comparison between NBI-B 1 images and squamous dysplasia with ASD (fig 8)}

We examined 67 abnormal fluorescence sites using high magnification bronchovideoscopy combined with NBI. The dotted vessels among the increased vessel growth and complex networks of tortuous vessels were observed at 18 abnormal fluorescence sites with NBI-Bl images, at which 14 $(78 \%)$ exhibited ASD. The negative dotted vessels among the vascular networks were at 49 abnormal fluorescence sites, at which 48 (98\%) were without ASD. There was a significant association between the frequency of dotted vessels by NBIBl imaging and pathologically confirmed ASD ( $p=0.002$ by Fisher's exact probability test).

\section{DISCUSSION}

In this study we used a method of high magnification bronchovideoscopy combined with NBI on a group of heavy smokers at high risk for lung cancer. Microvessels, vascular

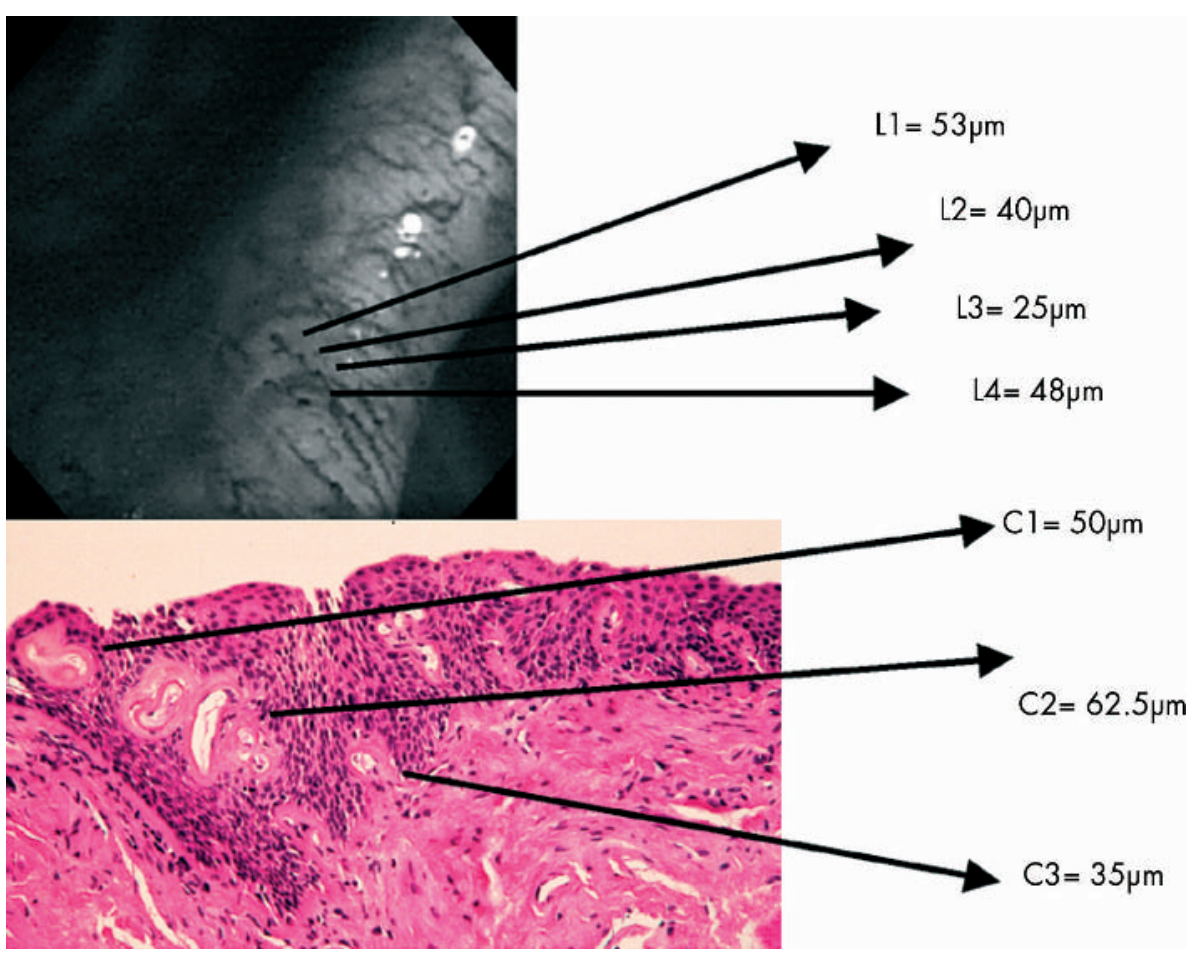

Figure 6 Comparison between dotted vessels observed by high magnification bronchovideoscopy combined with NBI and microscopic observation of capillary sized blood vessels projecting into dysplastic epithelium (ASD). 


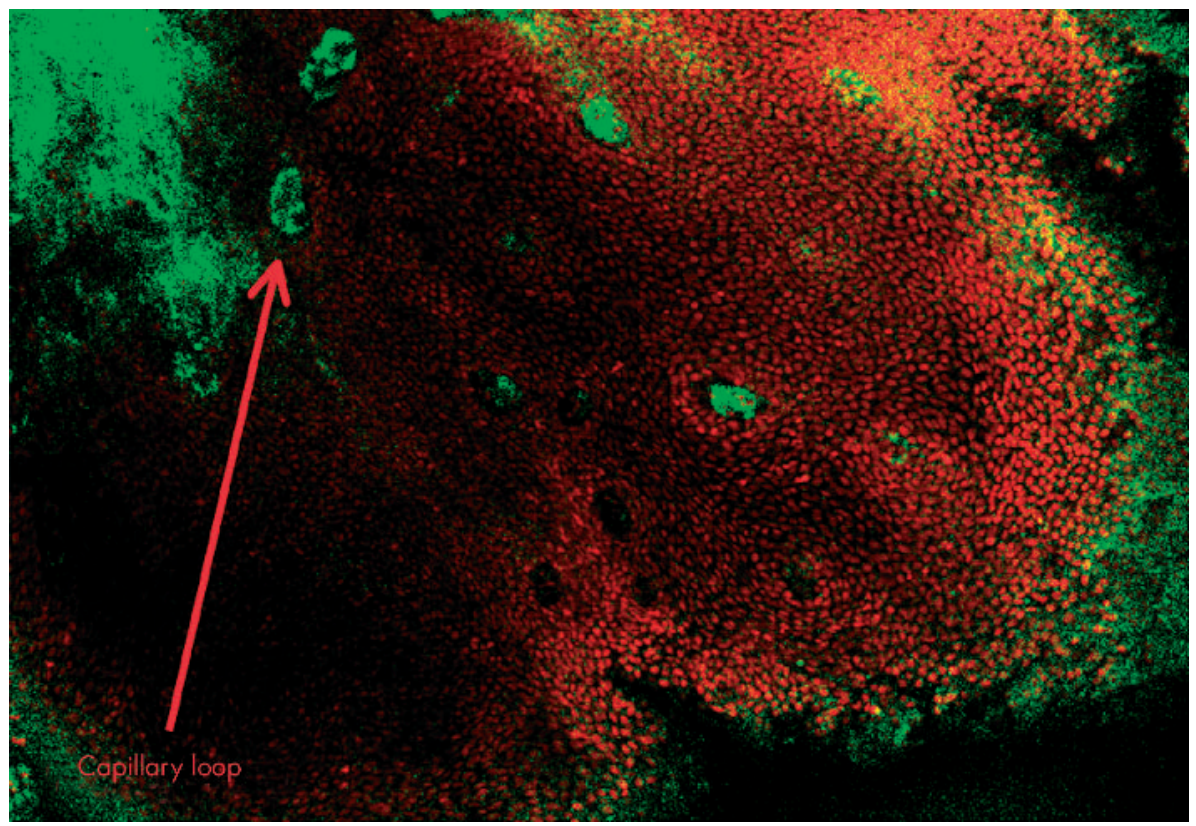

Figure 7 Dual immunolabelling by confocal laser scanning microscopy. Capillary blood vessels were clearly visualised as green fluorescence, while nuclei of dysplastic epithelium appeared orange in colour by confocal laser scanning microscopy.

networks of various grades, and dotted vessels were clearly observed in NBI-Bl images of ASD. In a previous study we showed that high magnification bronchovideoscopy, using a conventional RGB broadband filter on a RGB sequential videoscope system, facilitated the observation of microvascular networks in the bronchial mucosa. ${ }^{11}$ Our present study showed that bronchial mucosa microvascular networks could be distinctly observed in NBI-Bl images obtained using the new NBI filter that could not be observed using conventional RGB broadband filters, including conventional $\mathrm{B}$ images. Of the three NBI filters (NBI-B1, NBI-B2, and NBI-G) microvascular networks, including dotted vessels, were most clearly detected in NBI-Bl wavelengths. Tissue optical absorption and scattering properties are strongly wavelength dependent, so that blue light, which has a relatively short wavelength, typically reaches into shallow surfaces. ${ }^{13}$ The main chromophore in bronchial tissues in the visible wavelength range is haemoglobin, which has a maximum

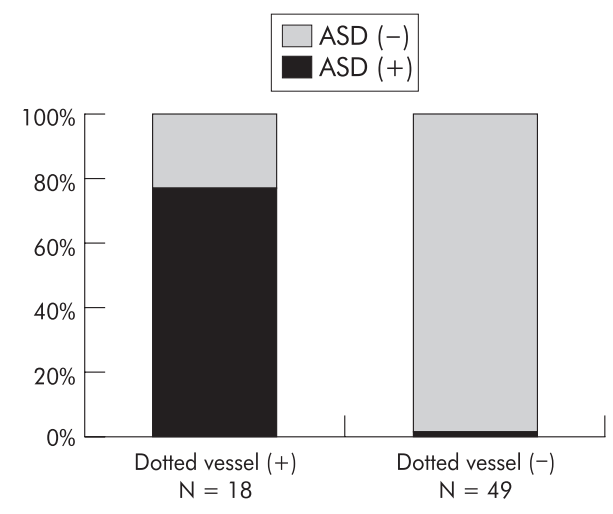

NBI-Blue 1 images

Figure 8 Comparison between NBI-Bluel (NBI-B1) images and squamous dysplasia with ASD. The dotted vessels were observed at 18 abnormal fluorescence sites with NBI-B1 images, at which 14 (78\%) exhibited ASD. The negative dotted vessels among the vascular networks were at 49 abnormal fluorescence sites, at which $48(98 \%)$ were without ASD. There was significant association between the frequency of dotted vessels by NBI-B1 imaging and pathologically confirmed ASD ( $p=0.002$ by Fisher's exact probability test). absorptive wavelength of approximately $415 \mathrm{~nm}$. As this is within the range of the new NBI-Bl filter, our new NBI instrument more accurately detected blood vessel structures than filters of other light wavelengths.

We previously reported that some of the vessels detected as scattered red dots, which may have the possibility of high magnification bronchovideoscopy with conventional white light, were capillary sized blood vessels in ASD. ${ }^{11}$ However, the diameter of the dotted vessels observed by NBI-BI imaging agreed with the values for ASD capillary blood vessel diameters obtained by pathological examination. As capillary blood vessels in ASD project into the dysplastic bronchial epithelium, the dotted vessels in the NBI-Bl images represented ASD capillary blood vessels. Thus, high magnification bronchovideoscopy combined with NBI is a new investigatory tool to detect capillary blood vessels in ASD lesions, and is able to demonstrate the onset of angiogenesis during multi-step carcinogenesis of the lung in heavy smokers at high risk for lung cancer.

LIFE fluorescence bronchoscopy has improved the recognition of bronchial dysplasia in patients at high risk for lung cancer. Hirsch et al reported that fluorescence bronchoscopy detected $75 \%$ of ASD cases. ${ }^{4}$ However, LIFE fluorescence bronchoscopy cannot distinguish between ASD and conventional squamous dysplasia (without ASD). Using our new imaging technique, we were able to distinguish ASD from another pre-neoplastic lesion. Prior to the introduction of fluorescence bronchoscopy, there was no means of locating areas of squamous dysplasia in the lower airways except for random bronchial biopsy. The results of our present study showed that our new method successfully detected ASD in addition to conventional squamous dysplasia. In the future, quantitative fluorescence imaging combined with fluorescence reflectance imaging and spectrofluorometry may also be helpful in improving the recognition of ASD. ${ }^{5} 17$

All participants with sputum cytology, suspicious or positive for malignancy, entered in this study were identified by a mass screening program for high lung cancer risk. Smoking history in pack years within our study group ranged from 25 to 155, with a mean of 54. All of the ASD lesions detected in our study were in heavy smoking patients, while ASD has not been detected in non smokers by our investigation or by Keith et $a l^{6}{ }^{6}$ indicating that ASD and 
smoking may be related. ${ }^{10}{ }^{18}$ Nicotine from cigarette smoking led to significant increases in endothelial cell vascular endothelial growth factor (VEGF) expression as shown by RT-PCR and Western blot analyses, ${ }^{19}$ and caused significantly increased serum VEGF levels and angiogenesis induction in a mouse model. ${ }^{20}$ In our investigation, the finding that ASD was detected in heavy smokers but not in non smokers suggests that nicotine may stimulate angiogenesis in human bronchial epithelium of the large airways.

Confocal laser scanning microscopy technology has enabled the visualisation of capillary network structures of various tissues, including tumour tissues. ${ }^{21-23}$ In experimental animal models of tumour angiogenesis, multiphoton laser scanning microscopy quantified angiogenesis with respect to different vessel types at different tumour depths. ${ }^{24}$ However, regarding pre-neoplastic lesions such as squamous dysplasia of bronchial epithelium, there have been no reports analysing angiogenic events using confocal laser scanning microscopy. In our study, using a fluorescence probe, capillary loops in the dysplastic epithelium of histopathologically confirmed ASD was detected by green fluorescence. Thus, confocal laser scanning microscopy technology is an appropriate method for demonstrating angiogenesis in pre-malignant bronchial epithelium.

That angiogenesis is essential for tumour growth was first recognised by Folkman. ${ }^{25}{ }^{26}$ In order to progress to a large size, incipient neoplasias must develop angiogenic capabilities. Several studies investigating the multiple step model of carcinogenesis in epithelial tumours have shown that angiogenesis is required in addition to molecular changes. ${ }^{27}$ An angiogenic switch appears to occur in pre-invasive lesions prior to invasive tumour formation in transgenic mouse models and human cancer pathogenesis studies. ${ }^{28} 29$ Neovascularization was evident in intraepithelial neoplasias II-III of cervical epithelium and breast carcinoma lesions by in situ immunochemistry, and other pre-invasive angiogenic lesions have also been documented in detail. ${ }^{28}{ }^{30}$ It has been suggested that fluorescence bronchoscopy could be applied to the investigation of bronchial pre-invasive lesions and multistep carcinogenesis. In this study, we employed the onset of angiogenesis in bronchial squamous dysplasia in vivo using high magnification bronchovideoscopy combined with a NBI system.

It may be possible to divide bronchial squamous dysplasia according to risk for developing squamous cell carcinoma. Whether or not ASD is an indicator of progression to squamous cell carcinoma remains undetermined. At the present time, the ongoing longitudinal follow up study for the presence of ASD would address this question. ${ }^{4}$ We need to document molecular pathogenesis of squamous dysplasias with and without angiogenesis, as well as chemoprevention studies using antiangiogenic therapies in heavy smokers. It is possible that in the near future angiogenesis inhibitors, either administered together or added to chemotherapy, radiotherapy, or other modalities, will become part of standard and effective anti tumour therapies.

In conclusion, high magnification bronchovideoscopy combined with NBI is a new investigatory tool to detect capillary blood vessels in ASD lesions, and may enable the discrimination between ASD and another pre-invasive bronchial lesion.

\section{ACKNOWLEDGEMENTS}

We wish to thank K Gono, Y Morizane, S Takehana (Olympus Optical Corp, Tokyo, Japan) and M Maekawa (Department of Anatomy and Developmental Biology, Graduate School of Medicine, Chiba University) for their technical assistance.

\section{Authors' affiliations}

K Shibuya, H Hoshino, M Chiyo, A lyoda, S Yoshida, Y Sekine, T lizasa, Y Saitoh, M Baba, T Fujisawa, Departments of Thoracic Surgery, Graduate School of Medicine, Chiba University, 1-8-1 Inohana, Chuo-ku, Chiba 260-8670, Japan

K Hiroshima, H Ohwada, Basic Pathology, Graduate School of Medicine, Chiba University, 1-8-1 Inohana, Chuo-ku, Chiba 260-8670, Japan

Supported, in part, by Grant-in-Aid for Scientific Research (C) 13671376 from the Japan Society for the Promotion of Science

\section{REFERENCES}

1 Lam S, Kennedy T, Unger M, et al. Localization of bronchial intraepithelial neoplastic lesions by fluorescence bronchoscopy. Chest 1998;113:696-702

2 George PJ. Fluorescence bronchoscopy for the early detection of lung cancer. Thorax 1999;54:180-183.

3 Shibuya K, Fujisawa T, Hoshino $\mathrm{H}$, et al. Fluorescence bronchoscopy in the detection of preinvasive bronchial lesions in patients with sputum cytology suspicious or positive for malignancy. Lung Cancer 2001;32:19-25.

4 Hirsch FR, Prindiville SA, Miller YE, et al. Fluorescence versus white-light bronchoscopy for detection of preneoplastic lesions: a randomized study J Natl Cancer Inst 2001;93:1385-1391.

5 Kennedy TC, Lam S, Hirsch FR. Review of recent advances in fluorescence bronchoscopy in early localization of central airway lung cancer. Oncologist 2001;6:257-262.

6 Keith RL, Miller YE, Gemmill RM, et al. Angiogenic squamous dysplasia in bronchi of individuals at high risk for lung cancer. Clin Cancer Res 2000;6: 1616-1625.

7 Franklin WA. Diagnosis of lung cancer: pathology of invasive and preinvasive neoplasia. Chest 2000;117:80S-89S

8 Fisseler-Eckhoff A, Rothstein D, Muller KM. Neovascularization in hyperplastic, metaplastic and potentially preneoplastic lesions of the bronchial mucosa. Virchows Arch 1996;429:95-100.

9 Fontanini G, Calcinai A, Boldrini L, et al. Modulation of neoangiogenesis in bronchial preneoplastic lesions. Oncol Rep 1999;6:813-817.

10 Gazdar AF, Minna JD. Angiogenesis and the multistage development of lung cancers. Clin Cancer Res 2000;6:1611-1612.

11 Shibuya K, Hoshino H, Chiyo M, et al. Subepithelial vascular patterns in bronchial dysplasias using a high magnification bronchovideoscope. Thorax 2002;57:902-907.

12 Cheong W, Prahl S, Welch A. A review of the optical properties of biological tissues. IEEE J. Quantum Electron 1990;26:2166-2185.

13 DaCosta RS, Wilson BC, Marcon NE. Light-induced fluorescence endoscopy of the gastrointestinal tract. Gastrointest Endosc Clin N Am 2000;10:37-69.

14 Smith M, Denninghoff K, Lompado A, et al. Effect of multiple light paths on retinal vessel oximetry. Applied optics 2000;39:1183-1193.

15 Prahl S. OMLC. omlc.ogi.edu/spectra/hemoglobin/index.html.

16 Travis WD CT, Corrin B, Shimosato $Y$, et al. Histological typing of lung and pleural tumours. In: World Health Organization, International Histological Classification of Tumours. 3rd ed. New York: Springer-Verlag, 1999.

17 Kusunoki Y, Imamura F, Uda H, et al. Early detection of lung cancer with laser-induced fluorescence endoscopy and spectrofluorometry. Chest 2000;118:1776-1782

18 Hiroshima K, lyoda A, Shibuya K, et al. Evidence of neoangiogenesis and an increase in the number of proliferating cells within the bronchial epithelium of smokers. Cancer 2002;95:1539-1545.

19 Conklin BS, Zhao W, Zhong DS, et al. Nicotine and cotinine up-regulate vascular endothelial growth factor expression in endothelial cells. Am J Pathol 2002;160:413-418.

20 Heeschen $C$, Jang JJ, Weis $M$, et al. Nicotine stimulates angiogenesis and promotes tumor growth and atherosclerosis. Nat Med 2001;7:833-839.

21 Hansen-Smith FM. Capillary network patterning during angiogenesis. Clin Exp Pharmacol Physiol 2000;27:830-835.

22 Itoh J, Yasumura K, Takeshita T, et al. Three-dimensional imaging of tumor angiogenesis. Anal Quant Cytol Histol 2000;22:85-90.

23 Jin E, Ghazizadeh M, Fujiwara M, et al. Angiogenesis and phenotypic alteration of alveolar capillary endothelium in areas of neoplastic cell spread in primary lung adenocarcinoma. Pathol Int 2001;51:691-700.

24 Brown EB, Campbell RB, Tsuzuki Y, et al. In vivo measurement of gene expression, angiogenesis and physiological function in tumors using multiphoton laser scanning microscopy. Nat Med 2001;7:864-868.

25 Folkman J. What is the evidence that tumors are angiogenesis dependent? J Natl Cancer Inst 1990;82:4-6.

26 Folkman J. Seminars in Medicine of the Beth Israel Hospital, Boston. Clinical applications of research on angiogenesis. N Engl J Med 1995;333:1757-1763.

27 Hirsch FR, Franklin WA, Gazdar AF, et al. Early detection of lung cancer: clinical perspectives of recent advances in biology and radiology. Clin Cancer Res $2001 ; 7: 5-22$.

28 Hanahan D, Folkman J. Patterns and emerging mechanisms of the angiogenic switch during tumorigenesis. Cell 1996;86:353-364.

29 Hanahan D, Weinberg RA. The hallmarks of cancer. Cell 2000;100:57-70.

30 Smith-McCune KK, Weidner N. Demonstration and characterization of the angiogenic properties of cervical dysplasia. Cancer Res 1994;54:800-804. 